\title{
Clinical assessment of the effectiveness of treatment for acneiform rash - skin toxicity of EGFR inhibitors
}

\author{
E.A. Shatokhina, L.S. Kruglova \\ Central State Medical Academy, Moscow, Russia
}

Introduction. Inhibitors of the epidermal growth factor receptor (EGFR) cause severe signs of acneinform rash, which may serve as a reason for changing the methods of cancer therapy, making the effective correction of this side effect especially important.

Methods. 35 patients with I-II grades of acneiform rash were split into 3 groups. All patients received systemic antibiotic therapy, $100 \mathrm{mg}$ of doxycycline twice a day for 10 days, and different topical medications: tacrolimus, metronidazole, and betamethasone valerate combined with fusidic acid. Assessment was performed using Acne Dermatology Index (ADI) and Dermatology Life Quality Index (DLQI).

Results. After the first week of systemic doxycycline therapy all groups have demonstrated significant regression in rash. Afterwards the first group, receiving treatment with tacrolimus cream, has demonstrated the weakest treatment response (patients were transferred to the 3rd group's treatment schedule after 1 month of therapy). Second group, receiving metronidazole gel, has demonstrated a greater treatment response. The first group, where patients received a combined cream containing betamethasone valerate and fusidic acid, has demonstrated the fastest regression in terms of both ADI and DLQI (fig. 1,2).

Fig. 1. Dynamics of Acne Dermatology Index (ADI, points) in patients suffering from acneinform rash before and after various methods of skin toxicity therapy.
Fig. 2. Dynamics of Dermatology Life Quality Index (DLQI) in patients suffering from acneinform rash before and after various methods of skin toxicity therapy
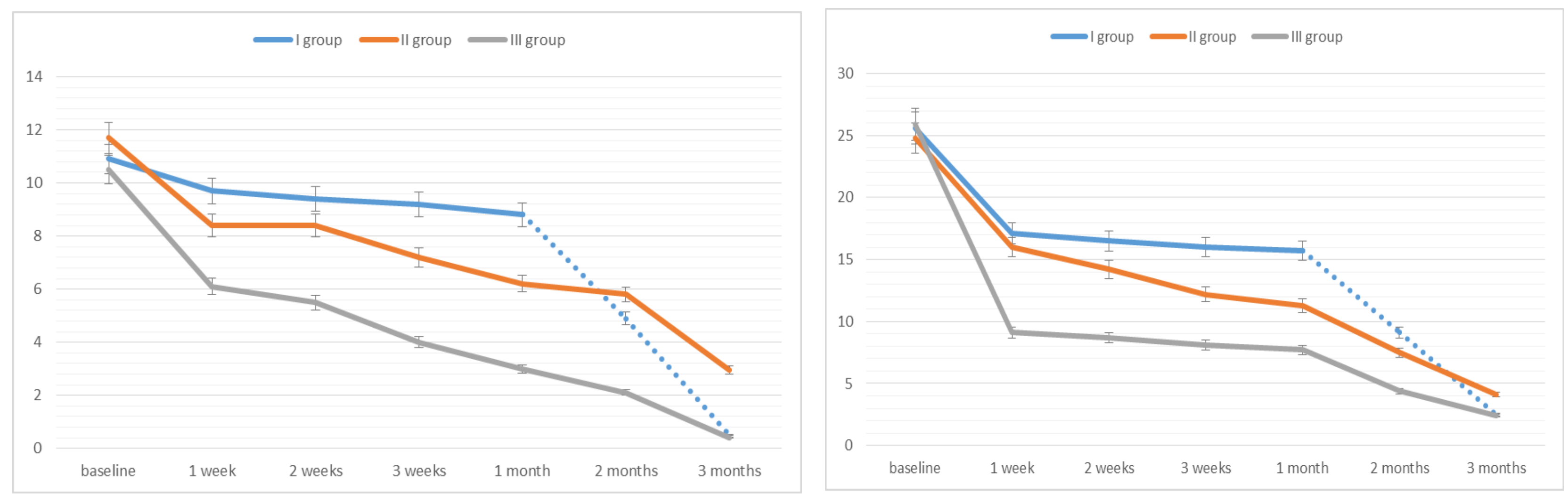

Conclusions. Systemic therapy with doxycycline at the early stages of I-II grade acneiform rash results in a significant clinical effect and prevents further deterioration in skin condition. Combined treatment including doxycycline and simultaneous application of topical medication containing $0.1 \%$ betamethasone valerate and $20 \%$ fusidic acid results in a faster and more prominent clinical effect as compared to other combinations: doxycycline with tacrolimus cream, and doxycycline with metronidazole gel. 